\title{
On the Spectrum of Energy in Turbulent Shear Flow
}

\author{
C. M. Tchen
}

\begin{abstract}
A turbulent flow with a given spatial pattern of mean motion invariant with time is treated. No solid boundary is considered. By means of the Fourier analysis of the NavierStokes equation, it is possible to obtain an equation of energy balance in spectral terms. This involves the following functions: Viscous dissipation, transfer, production, convection, and diffusion. The first two functions are common to the isotropic and homogeneous turbulence without mean motion. Assuming that eddies controlled by inhomogeneity are much larger than those controlled by anisotropy, stationary spectral laws are obtained for small eddies in the viscous and nonviscous ranges. The agreement between theory and experiment is shown.
\end{abstract}

\section{Introductory Remarks and Hypotheses on the Energy Distribution}

In the statistical theories of isotropic turbulence, the irregular motion of the fluid is described by some characteristic functions: the correlation function, and the spectral function. The statistical theories devoted to the analysis of the correlation function were developed by von Kármán, Burgers, and others [1]. ${ }^{1} \quad$ The study of the spectral function is based on the analysis of an irregular motion into harmonic components, as was developed by Burgers, for a model of turbulence [2]. The nonlinear process of the balance of turbulent energy is controlled by a transfer function, describing the transfer of energy from large eddies into small ones. Certain shapes of the transfer function were studied by Onsager [3], Obukhoff [4], von Weizäcker [5], and Heisenberg [6], all leading to a spectral law in agreement with the Kolmogoroff [7] law of locally isotropic turbulence for the nonviscous range. It seems that the spectral law in isotropic turbulence is well determined, at least for the above range of small eddies. In the following pages an attempt is made to study the spectral law of small eddies in turbulent shear flow with a given pattern of mean motion without solid boundaries. In addition to the viscous dissipation function and the transfer function, as occurring in isotropic turbulence without mean motion, we have to consider the production function, the convection function, and the diffusion function, which arise from the presence of a pattern of mean motion.

The Navier-Stokes equation applied to a turbulent flow can be divided into two equations: an equation governing the mean, or primary motion $U_{i}$, and an equation governing the life history of the turbulent velocity, or velocity fluctuation $u_{i}$. The equation for the turbulent velocity that interests us the most is, for an incompressible flow:

$$
\frac{\partial u_{i}}{\partial t}+\frac{\partial}{\partial x_{j}}\left(u_{i} U_{j}\right)=\nu \frac{\partial^{2} u_{i}}{\partial x_{j}^{2}}-\frac{\partial}{\partial x_{j}}\left(U_{i} u_{j}\right)-\frac{\partial}{\partial x_{j}}\left(u_{i} u_{j}-\overline{u_{i} u_{j}}+\frac{p}{\rho} \delta_{i j}\right) .
$$

The mean velocity $U_{i}$ and the velocity fluctuation $u_{i}$ must fulfill the equations of continuity

$$
\partial U_{i} / \partial x_{i}=0, \quad \partial u_{i} / \partial x_{i}=0 .
$$

The independent variables in the equations are the time $t$ and the coordinates $x_{i}, x_{j}$. Like the velocity, the pressure is decomposed into a mean pressure $P$, and a superposed pressure fluctuation $p . \quad \rho$ represents the density of the fluid and $\mu$ its viscosity, while $\nu=\mu / \rho . \quad \rho, \mu, \nu$ are treated as constant. The indices $i, j$, denote projection components. They are $i=1,2,3, j=1,2,3$. A summation is understood where the indices repeat. The bar over a quantity represents a spatial mean value.

We shall study the spectral distribution of the energy of the turbulent motion in a given field of primary motion. The interaction between the primary and the turbulent motion is controlled by terms such as $\partial\left(u_{i} U_{j}\right) / \partial x_{j}$, and $\partial\left(U_{i} u_{j}\right) \partial x_{j}$ in the hydrodynamical equation (1). According to this equation, the structure of the field at a given instant is characterized by three kinds of motions - a mean or primary motion, which gives rise to production, convection, and diffusion of turbulence; a turbulent motion, which possesses a transfer of energy; and a molecular motion playing the role of viscous dissipation. The three motions have their own geometrical patterns generally different in average scales. In order to fix our ideas, we can choose a large

1 Figures in brackets indicate the literature references at the end of this paper. 
region of size $R$, in which the primary motion shows certain geometrical patterns. At a given point we draw an element of volume of dimensions $2 X_{1}, 2 X_{2}, 2 X_{3}$, having several times the dimensions of the largest eddy to be included in the study. If we study the spectrum, or the energy carried by the eddies of different sizes contained in the element of volume, we can expect, according to the differential orders of the terms in the hydrodynamical equation, that the molecular action enters from the end of the small eddies, and the action of the primary motion from the end of the large eddies.

If we do not go down to the range of the molecular sizes, the action of the viscosity on the turbulent motion has a simple form, where the two motions are separated. On the other hand, if we do not go up to the very big eddies, and if the primary motion has a slowly varying pattern, or simple pattern, so that within the element of volume the velocity change can be considered constant and small, the action of the primary motion on the turbulent motion has also a simple form, where the two motions are separated without resonance. On the contrary, if the primary motion has a complexly varying pattern, or rich pattern, so that in the volume element are presented various scales of the patterns of the primary motion, and that certain small scales are comparable to the eddies of the turbulent motion, the two motions will interact with resonance and are not separable. Such circumstances will occur when the average scale of the primary motion, as depending on $U^{\prime} / U^{\prime \prime}, U^{\prime \prime} / U^{\prime \prime \prime}$, etc., according to the similarity theory, is small and becomes comparable to the scales of the eddies to be studied.

Let us isolate the finite element of volume from its surroundings and study first, by means of an elementary method, the balance of energy per unit volume among the different eddies. For this purpose, let $k$ be the wave-number magnitude, $v_{k}$ the velocity, and $l \sim 1 / k$ the mixing length. The vorticity of the turbulent motion can be represented by $v_{k} / l$. The vorticity presented in the pattern of the primary motion, averaged over the element, is supposed to be given, and is denoted by $U^{\prime}$.

Let $S_{k}$ be the flow of energy from larger eddies (larger than the eddy $k$ ) to smaller eddies (smaller than the eddy $k$ ). Such a flow of energy corresponds to a dissipation due to the following exchanges of momentum: between the molecular motion and the turbulent motion, between the small and large eddies, and between the turbulent motion and the primary motion. According to Prandtl and Boussinesq, it is known that the dissipation is the product of the viscosity with the square of the vorticity.

For the dissipation of molecular origin, we can write $\mu v_{k}^{2} / l^{2}$, where $\mu$ is the coefficient of viscosity due to molecular agitations. For the dissipation of turbulent origin, that is, the dissipation due to the exchange of momentum between the eddies of the turbulent motion itself, and between the turbulent motion and the primary motion, we can write

$$
\text { turbulent dissipation }=\rho \nu_{k} \cdot \text { compound square velocity. }
$$

Here $\nu_{k}$ is the turbulent viscosity of Heisenberg $\nu_{k}=v_{k} l$. If there is no resonance between the primary motion and the turbulent motion, the compound square vorticity is formed by the following independent terms:

$$
\frac{v_{k}^{2}}{l^{2}}+U^{\prime 2}
$$

the interaction term $\left(v_{k} / l\right) U^{\prime}$ being dropped out. If there is strong resonance between the two motions, the independent term $U^{\prime 2}$ must be dropped out, because any primary vorticity field that is able to create momentum exchange with eddies must be coupled to the turbulent vorticity. Thus the compound square vorticity becomes in this case

$$
\frac{v_{k}^{2}}{l^{2}}+\frac{v_{k}}{l} U^{\prime}
$$

Hence the equation of total dissipation is

$$
S_{k}=\mu \frac{v_{k}^{2}}{l^{2}}+\rho v_{k} l .\left\{\frac{v_{k}^{2}}{l^{2}}+\left\langle\frac{U^{\prime 2}}{l} U_{k}\right\}\right. \text { no resonance. }
$$

The case of intermediate resonance is not investigated here.

A spectral function $F(k)$ can be introduced such that $\rho F(k) d k$ is the turbulent energy between $k$ and $k+d k$. Then by means of dimensional reasoning, the dissipation equation can be put 
in spectral terms as follows:

$$
\begin{aligned}
\frac{S_{k}}{\rho}= & 2 \nu \int_{0}^{k} d k^{\prime} k^{\prime 2} F\left(k^{\prime}\right)+2 \kappa \int_{k}^{\infty} d k^{\prime \prime} \sqrt{\frac{F\left(k^{\prime \prime}\right)}{k^{\prime \prime}}} \int_{0}^{k} d k^{\prime} k^{\prime 2} F\left(k^{\prime}\right)+ \\
& \kappa^{\prime} \int_{k}^{\infty} d k^{\prime \prime} \sqrt{\frac{F\left(k^{\prime \prime}\right)}{k^{\prime \prime}}} U^{\prime} \cdot \frac{U^{\prime}}{\frac{\kappa^{\prime \prime}}{\kappa^{\prime}}}\left[2 \int_{0}^{k} d k^{\prime} k^{\prime 2} F\left(k^{\prime}\right)\right]^{\frac{1}{2}} \text { no resonance }
\end{aligned}
$$

where $\kappa, \kappa^{\prime}, \kappa^{\prime \prime}$ are numerical constants to be determined by experiments.

We shall study the spectral laws in the equilibrium range of spectrum, which, as defined by Heisenberg, is the range controlled by a constant flow of energy $S_{k}$, independent of $k$. In the equilibrium range, two subranges are defined: the nonviscous, or inertial, subrange, as characterized by $v_{k} / k \nu \gg 1$, and the viscous subrange, as characterized by $v_{k} / k \nu \ll 1$. For the nonviscous subrange we obtain

$$
v \sim k^{-1 / 3}, \quad \text { or } \quad F \sim k^{-5 / 3} \quad \text { for small } U^{\prime} \text { and no resonance; }
$$

and

$$
v_{k} \sim \text { constant }, \quad \text { or } \quad F \sim k^{-1} \quad \text { for large } U^{\prime} \text { and strong resonance. }
$$

For the viscous subrange we obtain

$$
v_{k} \sim k^{-3}, \quad \text { or } \quad F \sim k^{-7} \quad \text { for arbitrary } U^{\prime} \text { and resonance. }
$$

The spectral law $k^{-7}$ is not expected to go down to molecular scales, as then the strong interference between the molecular motion and the turbulent motion will alter the above simple forms of the molecular and turbulent dissipations.

The above elementary study of the spectrum of turbulence is based on the considerations of the different forms of the exchange of momentum within an isolated volume element. Actually, the volume element is not isolated, and the general character of the field from one element to another may vary. Under those circumstances we may expect that the general spectrum depends on the position of the element analyzed, and that there exists an exchange of momentum between the element chosen and its neighbors, in the forms of convection and diffusion. However, the latter forms of exchange, called inhomogeneous exchange, have scales comparable to the dimensions of the volume elements and thus affect predominantly the large eddies rather than the eddies in the equilibrium range in which we are interested for the present. We shall return to the discussions of convection and diffusion in sections 3 and 4 . We conclude that in the study of the equilibrium range of spectrum, we can analyze the inhomogeneous field as follows:

1. We choose a finite element of volume $2 X_{1} \cdot 2 X_{2} \cdot 2 X_{3}$, which is of the order of several times the scales of the largest eddy of the equilibrium range. In the element of volume a turbulent motion is superposed to a primary motion exhibiting certain geometrical patterns. Both motions can be analyzed into Fourier components by the same method as applied to a homogeneous field.

2. The inhomogeneity of the general field creates an inhomogeneous exchange of momentum between the chosen element of volume and its neighbors, resulting in convection and diffusion. Such an exchange of large scales is not important to the study of eddies in the equilibrium range (small eddies). Thus it can be estimated approximately and added to the homogeneous exchange as small corrections.

\section{Harmonic Analysis of a Turbulent Motion}

The results predicted in section 1 by means of elementary considerations of energy distribution can be obtained by a harmonic analysis of the hydrodynamical equations. Such an analysis will reveal some important details of the exchange mechanism and of the energy balance, which are rather obscure in the elementary considerations.

The applications of Fourier analysis to a turbulent field has the difficulty that Fourier integrals can be defined only for functions that vanish at infinity in such a way that their squares take a finite integrable value. To overcome this difficulty, one usually assumes in the harmonic analysis of turbulence that we can restrict to the values of the function within a finite domain, say a finite element of volume $2 X_{1} \cdot 2 X_{2} \cdot 2 X_{3}$ taken at a point $x_{1}, x_{2}, x_{3}$ as center, while outside 
this domain the function is replaced by zero. ${ }^{2}$ For the purpose of the analysis of turbulence, the Fourier integral of the turbulent velocity in such a finite domain will be sufficiently representative of the energy distribution of eddies smaller than the element. The amplitude functions of the Fourier coefficients are then obtained from

$$
\begin{aligned}
a_{i}(\boldsymbol{k}) & =\frac{1}{(2 \pi)^{3}} \int_{x-\boldsymbol{X}}^{x+\boldsymbol{X}} d \boldsymbol{x}^{\prime} u_{i}\left(\boldsymbol{x}^{\prime}\right) \mathrm{e}^{-\imath \boldsymbol{k} \cdot \boldsymbol{x}^{\prime}} \\
A_{i}(\boldsymbol{k}) & =\frac{1}{(2 \pi)^{3}} \int_{x-\boldsymbol{X}}^{x+\boldsymbol{X}} d \boldsymbol{x}^{\prime} U_{i}\left(\boldsymbol{x}^{\prime}\right) \mathrm{e}^{-\imath \boldsymbol{k} \cdot \boldsymbol{x}^{\prime}} \\
c(\boldsymbol{k}) & =\frac{1}{(2 \pi)^{3}} \int_{x-\boldsymbol{X}}^{x+\boldsymbol{X}} d \boldsymbol{x}^{\prime} p\left(\boldsymbol{x}^{\prime}\right) \mathrm{e}^{-\imath \boldsymbol{k} \cdot \boldsymbol{x}^{\prime}}
\end{aligned}
$$

with the equations of continuity

$$
k_{i} a_{i}(\boldsymbol{k})=0, \quad k_{i} A_{i}(\boldsymbol{k})=0 .
$$

Here $u_{i}, U_{\imath}, p$ are real quantities representing the turbulent velocity components, the mean velocity components, and the turbulent pressure, respectively, $a_{i}(\boldsymbol{k}), A_{i}(\boldsymbol{k}), c(\boldsymbol{k})$ are the corresponding Fourier coefficients at the wave-number vector $\boldsymbol{k}(\boldsymbol{k}=2 \pi /$ wavelength $)$, and are complex quantities, with $a_{i}^{*}(\boldsymbol{k})=a_{i}(-\boldsymbol{k})$, etc. An asterisk over a quantity denotes its conjugate, and $\iota=\sqrt{-1}$. The following symbol for the volume integral is used:

$$
\int_{a}^{b} d \boldsymbol{x}^{\prime}=\int_{a_{1}}^{b_{1}} \int_{a_{2}}^{b_{2}} \int_{a_{3}}^{b_{3}} d x_{1}^{\prime} d x_{2}^{\prime} d x_{3}^{\prime}
$$

The actual change of $u_{i}$ and $p$ in the course of time is resolved into the change of the Fourier coefficients $a_{i}$ and $c$ with the time. Provisionally, we restrict to the single instant. The analysis in a finite volume renders the Fourier coefficients bounded. Outside and on the boundary of the finite volume $\left(\boldsymbol{x}^{\prime} \leq \boldsymbol{x}-\boldsymbol{X}, \boldsymbol{x}^{\prime} \geq \boldsymbol{x}+\boldsymbol{X}\right)$, the function $u_{i}\left(\boldsymbol{x}^{\prime}\right)$ and its derivative $\partial u_{i}\left(\boldsymbol{x}^{\prime}\right) / \partial x_{j}^{\prime}$ are supposed to vanish; so also do $p\left(\boldsymbol{x}^{\prime}\right)$ and $U_{i}\left(\boldsymbol{x}^{\prime}\right)$. Such functions are called truncated functions.

To show the significance of the limit $\boldsymbol{X}$, we form:

$$
u_{i}(\boldsymbol{x}) u_{j}(\boldsymbol{x}+\boldsymbol{r})=\int_{-\infty}^{+\infty} d \boldsymbol{k}^{\prime} \int_{-\infty}^{+\infty} d \boldsymbol{k}^{\prime \prime} a_{i}\left(\boldsymbol{k}^{\prime}\right) a_{j}\left(-\boldsymbol{k}^{\prime \prime}\right) \mathrm{e}^{\iota\left(\boldsymbol{k}^{\prime}-\boldsymbol{k}^{\prime \prime}\right) \cdot \boldsymbol{x}-\imath \boldsymbol{k}^{\prime \prime} \cdot \boldsymbol{r}}
$$

The mean value $\overline{u_{i}(\boldsymbol{x}) u_{j}(\boldsymbol{x}+\boldsymbol{r})}$ is obtained by integrating with respect to $\boldsymbol{x}$ from $-\boldsymbol{X}^{\prime}$ to $+\boldsymbol{X}^{\prime}$, where $\boldsymbol{X}^{\prime}>\boldsymbol{X}$. We have

$$
\begin{aligned}
\overline{u_{i}(\boldsymbol{x}) u_{j}(\boldsymbol{x}+\boldsymbol{r})}= & \frac{1}{X_{1} X_{2} X_{3}} \int_{-\infty}^{+\infty} d \boldsymbol{k}^{\prime} \int_{-\infty}^{+\infty} d \boldsymbol{k}^{\prime \prime} \mathrm{e}^{-\imath \boldsymbol{k}^{\prime \prime} \cdot r} a_{i}\left(\boldsymbol{k}^{\prime}\right) a_{j}\left(-\boldsymbol{k}^{\prime \prime}\right) . \\
& \frac{\left(\sin k_{1}^{\prime}-k_{1}^{\prime \prime}\right) X_{1}^{\prime}}{k_{1}^{\prime}-k_{1}^{\prime \prime}} \frac{\sin \left(k_{2}^{\prime}-k_{2}^{\prime \prime}\right) X_{2}^{\prime}}{k_{2}^{\prime}-k_{2}^{\prime \prime}} \frac{\sin \left(k_{3}^{\prime}-k_{3}^{\prime \prime}\right) X_{3}^{\prime}}{k_{3}^{\prime}-k_{3}^{\prime \prime}},
\end{aligned}
$$

which for $\boldsymbol{X}^{\prime} \rightarrow \infty$ leads to

$$
\overline{u_{i}(\boldsymbol{x}) u_{j}(\boldsymbol{x}+\boldsymbol{r})}=\frac{\pi^{3}}{X_{1} X_{2} X_{3}} \int_{-\infty}^{+\infty} d \boldsymbol{k} a_{i}(\boldsymbol{k}) a_{j}(-\boldsymbol{k}) \mathrm{e}^{-\imath \boldsymbol{k} \cdot \boldsymbol{r}} \cdot
$$

Since $\overline{u_{i}(\boldsymbol{x}) u_{j}(\boldsymbol{x}+\boldsymbol{r})}$ must be independent of $\boldsymbol{X}$, it follows that the absolute value of the amplitude function must be proportional to $\left(X_{1} X_{2} X_{3}\right)^{1 / 2}$. As $a_{i}$ is obtained by the summation of a large number of variables like $u_{i}\left(\boldsymbol{x}^{\prime}\right) e^{\iota \boldsymbol{k} \cdot \boldsymbol{x}^{\prime}}$ according to (3), the fact that its absolute value is proportional to the square root of the volume must be related to the same results found in problems of radom walk, even though the paths may be correlated [10].

2 To avoid discontinuities that may arise by such a method at the edges of the elementary volume, we may instead multiply the function by a certain function that is unity within the volume, and decays rapidly to zero beyond the volume. 
If we take $r=0$ and $j=i$, we find

$$
\overline{u_{i}^{2}}=\frac{\pi^{3}}{X_{1} X_{2} X_{3}} \int_{-\infty}^{+\infty} d \boldsymbol{k} a_{i}(\boldsymbol{k}) a_{i}(-\boldsymbol{k})
$$

Instead of extending the integral over the whole $k$-space, we can integrate over a spherica! shell of radii between $k$ and $k+d k$, and we find

$$
2 F(k)=\frac{\pi^{3}}{X_{1} X_{2} X_{3}} \int_{0}^{2 \pi} d \varphi \int_{0}^{\pi} d \theta \sin \theta k^{2} a_{i}(\boldsymbol{k}) a_{i}(-\boldsymbol{k}) \quad \text { with } \quad 2 \int_{0}^{\infty} d k^{\prime} F\left(k^{\prime}\right)=\overline{u_{\imath}^{2}}
$$

Here $k^{2} \sin \theta d \theta d \varphi$ is the volume element with polar angles $\theta, \varphi$, and $F(k) d k$ is the energy associated with the wave numbers ranged between $k$ and $k+d k$. $F(k)$ will be referred to customarily as spectral function of turbulent energy. Also we can define a spectral function of the turbulent shear stress $F_{i j}(k)$ such that

$2 F_{i j}(k)=\frac{\pi^{3}}{X_{1} X_{2} X_{3}} \int_{0}^{2 \pi} d \varphi \int_{0}^{\pi} d \theta \sin \theta k^{2} \frac{1}{2}\left[a_{i}(\boldsymbol{k}) a_{j}(-\boldsymbol{k})+a_{i}(-\boldsymbol{k}) a_{j}(\boldsymbol{k})\right]$ with $2 \int_{0}^{\infty} d k^{\prime} F_{i j}\left(k^{\prime}\right)=\overline{u_{i} u_{j}}$.

By a Fourier transform of (1) term by term, we have

$$
\begin{aligned}
\frac{\partial a_{i}(\boldsymbol{k})}{\partial t}+ & \int_{-\infty}^{+\infty} d \boldsymbol{n} \iota n_{j} a_{i}(\boldsymbol{n}) A_{j}(\boldsymbol{k}-\boldsymbol{n})=-\nu k^{2} a_{i}(\boldsymbol{k})-\int_{-\infty}^{+\infty} d \boldsymbol{n} \iota n_{j} A_{i}(\boldsymbol{n}) a_{j}(\boldsymbol{k}-\boldsymbol{n})- \\
& \int_{-\infty}^{+\infty} d \boldsymbol{n} \iota n_{j} a_{i}(\boldsymbol{n}) a_{j}(\boldsymbol{k}-\boldsymbol{n})-\frac{1}{\rho} \iota k_{i} c(\boldsymbol{k}) \text {-inhomogeneous terms, }
\end{aligned}
$$

where the inhomogeneous terms arise from the inhomogeneous exchange as explained in section 1 and to be determined in sections 3 and 4 . In the above equation $c$ can be eliminated by its value obtained from the above equation multiplied by $k_{i}$, subjected to the conditions of continuity (4). The transformed equation is then

$$
\begin{aligned}
\frac{\partial a_{i}(\boldsymbol{k})}{\partial t}=-\nu k^{2} a_{i}(\boldsymbol{k})- & \int_{-\infty}^{+\infty} d \boldsymbol{n} \iota n_{j} a_{j}(\boldsymbol{k}-\boldsymbol{n})\left[a_{i}(\boldsymbol{n})-\frac{k_{i} k_{s}}{k^{2}} a_{s}(\boldsymbol{n})\right]- \\
& \int_{-\infty}^{+\infty} d \boldsymbol{n} \iota n_{j} a_{j}(\boldsymbol{k}-\boldsymbol{n})\left[A_{i}(\boldsymbol{n})-\frac{k_{i} k_{s}}{k^{2}} A_{s}(\boldsymbol{n})\right]- \\
& \int_{-\infty}^{+\infty} d \boldsymbol{n} \iota n_{j} A_{j}(\boldsymbol{k}-\boldsymbol{n})\left[a_{i}(\boldsymbol{n})-\frac{k_{i} k_{s}}{k^{2}} a_{s}(\boldsymbol{n})\right]-\text { inhomogeneous terms. }
\end{aligned}
$$

An equation describing the rate of change of the energy distribution can be obtained by forming the differential quotient $(\partial / \partial t) a_{i}(\boldsymbol{k}) a_{i}(-\boldsymbol{k})$ as follows:

$\frac{\partial}{\partial t} a_{i}(\boldsymbol{k}) a_{i}(-\boldsymbol{k})=-2 \nu k^{2} a_{i}(\boldsymbol{k}) a_{i}(-\boldsymbol{k})-$

$$
\begin{aligned}
& \int_{-\infty}^{+\infty} d \boldsymbol{n} \iota n_{j} a_{i}(\boldsymbol{n})\left[a_{j}(\boldsymbol{k}-\boldsymbol{n}) a_{i}(-\boldsymbol{k})+a_{j}(-\boldsymbol{k}-\boldsymbol{n}) a_{i}(\boldsymbol{k})\right]- \\
& \int_{-\infty}^{+\infty} d \boldsymbol{n} \iota n_{j} A_{i}(\boldsymbol{n})\left[a_{j}(\boldsymbol{k}-\boldsymbol{n}) a_{i}(-\boldsymbol{k})+a_{j}(-\boldsymbol{k}-\boldsymbol{n}) a_{i}(\boldsymbol{k})\right]- \\
& \int_{-\infty}^{+\infty} d \boldsymbol{n} \iota n_{j} a_{i}(\boldsymbol{n})\left[A_{j}(\boldsymbol{k}-\boldsymbol{n}) a_{i}(-\boldsymbol{k})+A_{j}(-\boldsymbol{k}-\boldsymbol{n}) a_{i}(\boldsymbol{k})\right] \text {-inhomogeneous terms. }
\end{aligned}
$$

The physical meaning of the equation for energy distribution (7) can be compared with the following equation for over-all balance obtained by multiplying (1) by $u_{i}$ and taking the spatial mean values as follows:

$$
\frac{\partial}{\partial t} \frac{\overline{u_{i}^{2}}}{2}=-\nu \overline{\left(\frac{\partial u_{i}}{\partial x_{j}}\right)^{2}}-\overline{u_{i} u_{j}} \frac{\partial U_{i}}{\partial x_{j}}-\frac{\partial}{\partial x_{j}}\left(\overline{\frac{u_{i}^{2}}{2}} U_{j}\right)+\nu \frac{\partial^{2}}{\partial x_{i}^{2}} \frac{\overline{u_{i}^{2}}}{2}-\frac{\partial}{\partial x_{j}}\left[\overline{u_{j}\left(\frac{u_{i}^{2}}{2}+\frac{p}{\rho}\right)}\right]
$$


Equation (7) represents the balance of energy associated with the wave number $k$, while eq (7a) represents the balance of energy covering the whole spectrum. The correspondence between terms of the two equations is as follows:

(a) Rate of change of energy:

$$
\frac{1}{2} \frac{\pi^{3}}{X_{1} X_{2} X_{3}} \int_{-\infty}^{+\infty} d \boldsymbol{k} \frac{\partial}{\partial t} a_{i}(\boldsymbol{k}) a_{i}(-\boldsymbol{k})=\frac{\partial}{\partial t} \frac{\overline{u_{i}^{2}}}{2}
$$

For a stationary flow these two terms vanish.

(b) Dissipation by viscosity:

$$
\frac{1}{2} \frac{\pi^{3}}{X_{1} X_{2} X_{3}} \int_{-\infty}^{+\infty} d \boldsymbol{k} 2 \nu k^{2} a_{i}(\boldsymbol{k}) a_{i}(-\boldsymbol{k})=\nu\left(\overline{\left(\mathrm{\partial} u_{i} / \partial x_{\imath}\right)^{2}}\right.
$$

(c) Transfer of energy from eddies to eddies:

$$
-\int_{-\infty}^{+\infty} d \boldsymbol{n} \iota n_{j} a_{i}(\boldsymbol{n})\left[a_{j}(\boldsymbol{k}-\boldsymbol{n}) a_{i}(-\boldsymbol{k})+a_{j}(-\boldsymbol{k}-\boldsymbol{n}) a_{i}(\boldsymbol{k})\right] .
$$

This term which figures in (7) has no corresponding term in (7a), because the total transfer must vanish for the whole spectrum.

(d) Production of turbulent energy by shearing stresses:

$$
\frac{1}{2} \frac{\pi^{3}}{X_{1} X_{2} X_{3}} \int_{-\infty}^{+\infty} d \boldsymbol{k} \int_{-\infty}^{+\infty} d \boldsymbol{n} \iota n_{j} A_{i}(\boldsymbol{n})\left[a_{j}(\boldsymbol{k}-\boldsymbol{n}) a_{i}(-\boldsymbol{k})+a_{j}(-\boldsymbol{k}-\boldsymbol{n}) a_{i}(\boldsymbol{k})\right]=\overline{u_{i} u_{j}} \frac{\partial U_{i}}{\partial x_{j}} .
$$

(e) Convection of turbulent energy by interaction with mean motion:

$$
\frac{1}{2} \frac{\pi^{3}}{X_{1} X_{2} X_{3}} \int_{-\infty}^{+\infty} d \boldsymbol{k} \int_{-\infty}^{+\infty} d \boldsymbol{n} \iota n_{j} a_{i}(n)\left[A_{j}(\boldsymbol{k}-\boldsymbol{n}) a_{i}(-\boldsymbol{k})+A_{j}(-\boldsymbol{k}-\boldsymbol{n}) a_{i}(\boldsymbol{k})\right]=\frac{\partial}{\partial x_{j}}\left(\overline{\frac{u_{i}^{2}}{2}} U_{j}\right) .
$$

(f) Inhomogeneous transport: This is the part of convection from the inhomogeneity of the field. Thus in (7a) the terms

$$
\frac{\partial}{\partial x_{j}} \frac{\overline{u_{i}^{2}}}{2}, \quad \frac{\partial^{2}}{\partial x_{j}^{2}} \frac{\overline{u_{i}^{2}}}{2}, \quad \frac{\partial}{\partial x_{j}}\left[\overline{u_{j}\left(\frac{u_{i}^{2}}{2}+\frac{p}{\rho}\right)}\right]
$$

correspond to the inhomogeneous terms of (7), to be determined in sections 3 and 4 . For a stationary condition the left-hand side of (7) must vanish. Integration between $k$ and $\infty$ gives

$$
2 \nu \int_{0}^{\infty} d k^{\prime} k^{\prime 2} F\left(k^{\prime}\right)=2 \nu \int_{0}^{k} d k^{\prime} k^{\prime 2} F\left(k^{\prime}\right)+W(k, \infty)+\psi(k, \infty)+C(k, \infty)+K(k, \infty) .
$$

This equation expresses the equilibrium in the exchange of energy. The left-hand side is the total viscous dissipation covering the whole spectrum. It is the total flow of energy. It is independent of $k$ and will be represented by $\epsilon$. The first term on the right-hand side gives the loss of energy through viscous dissipation associated with wave numbers not exceeding $k$. The other four terms give the flow of energy from this part of the spectrum through interaction with the remainder of the spectrum, respectfully, in the forms of transfer, production, convection, and diffusion, as to be obtained by integrating the corresponding terms of (7) for wave numbers not exceeding $k$.

\section{Transfer, Production, and Convection of Turbulence}

For the transfer function $W(k, \infty)$, we assume the Heisenberg formula [6].

$$
W(k, \infty)=\kappa \int_{k}^{\infty} d k^{\prime \prime} \sqrt{\frac{F\left(k^{\prime \prime}\right)}{k^{\prime \prime}}} 2 \int_{0}^{k} d k^{\prime} k^{\prime 2} F\left(k^{\prime}\right),
$$


where $\kappa$ is a numerical constant. The justification of the dissipative character of the transfer and hence of the Heisenberg theory, can be sought from the transport processes by turbulence on the basis of the hydrodynamical equations. We shall return to this problem in a subsequent communication.

For the production function $\Psi(k, \infty)$, we assume a gradient of $U_{1}$ in the $x_{2}$ direction, as expressed by $\partial U_{1} / \partial x_{2}$, all other gradients being negligible, and distinguish the case of no resonance and strong resonance, depending on the scale of the primary motion, as mentioned in section 1.

(a) When there is negligible resonance between the mean and the turbulent motions, we can neglect $n$ compared to $k$ in the following production term:

$$
\int_{-\infty}^{+\infty} d \boldsymbol{n} \iota n_{2} A_{1}(\boldsymbol{n})\left[a_{2}(\boldsymbol{k}-\boldsymbol{n}) a_{1}(-\boldsymbol{k})+a_{2}(-\boldsymbol{k}-\boldsymbol{n}) a_{1}(\boldsymbol{k})\right] \simeq\left[a_{1}(-\boldsymbol{k}) a_{2}(\boldsymbol{k})+a_{1}(\boldsymbol{k}) a_{2}(-\boldsymbol{k})\right] \cdot U^{\prime}
$$

where $^{2}$

$$
\int_{-\infty}^{+\infty} d \boldsymbol{n} \iota n_{2} A_{1}(\boldsymbol{n})=U^{\prime}
$$

is the magnitude of the average vorticity of the mean motion for a quickly dropping spectrum $A_{1}(\boldsymbol{n})$. Integration of the production term over the wave numbers of magnitudes between $k$ and $\infty$ gives

$$
\Psi(k, \infty)=2 \int_{k}^{\infty} d k^{\prime} F_{12}\left(k^{\prime}\right) \cdot U^{\prime}
$$

If, according to Prandtl and Broussinesq [8, 9,], we write

$$
2 \int_{k}^{\infty} d k^{\prime} F_{12}\left(k^{\prime}\right)=\kappa^{\prime} \int_{k}^{\infty} d k^{\prime \prime} \sqrt{\frac{F\left(k^{\prime \prime}\right)}{k^{\prime \prime}} \cdot U^{\prime}}
$$

where $k^{\prime} \int_{k}^{\infty} d k^{\prime \prime} \sqrt{F\left(k^{\prime \prime}\right) / k^{\prime \prime}}$ is a turbulent viscosity, and $\kappa^{\prime}$ is a numerical constant, eq (10) is reduced to

$$
\Psi(k, \infty)=\kappa^{\prime} \int_{k}^{\infty} d k^{\prime \prime} \sqrt{\frac{F\left(k^{\prime \prime}\right)}{k^{\prime \prime}}} \cdot U^{\prime 2} \text {, for negligible resonance. }
$$

(b) When there is strong resonance between the mean and turbulent motions as described in section 1 , in such a way that we cannot separate them a priori by the method used in (a), a new method has to be devised in order to formulate the production function $\Psi$. Integration of $(7)$ outside the sphere of radius $k$ gives the transfer and production functions as follows:

$$
\begin{aligned}
& -W(k, \infty)=\frac{\pi^{3}}{X_{1} X_{2} X_{3}} \int_{k}^{\infty} d \boldsymbol{k}^{\prime \prime} \int_{0}^{\infty} d \boldsymbol{n} \iota \boldsymbol{n}_{j} a_{i}(\boldsymbol{n}) a_{j}\left(\boldsymbol{k}^{\prime \prime}-\boldsymbol{n}\right) a_{i}\left(-\boldsymbol{k}^{\prime \prime}\right)+\text { conjugate } \\
& -\Psi(k, \infty)=\frac{\pi^{3}}{X_{1} X_{2} X_{3}} \int_{k}^{\infty} d \boldsymbol{k}^{\prime \prime} \int_{0}^{\infty} d \boldsymbol{n} \iota \boldsymbol{n}_{j} A_{i}(\boldsymbol{n}) a_{j}\left(\boldsymbol{k}^{\prime \prime}-\boldsymbol{n}\right) a_{i}\left(-\boldsymbol{k}^{\prime \prime}\right)+\text { conjugate. }
\end{aligned}
$$

${ }^{2}$ By applying the Fourier formula (3a), the amplitude function of the vorticity is

$$
\iota n_{2} A_{1}\left(n_{2}\right)=\frac{1}{2 \pi} \int_{-X_{2}}^{+X_{2}} d x_{2} \frac{\partial U_{1}}{\partial x_{2}} e^{-\iota n_{2} x_{2}}
$$

and

$$
\int_{-\infty}^{+\infty} d n_{2} \iota n_{2} A_{1}\left(n_{2}\right)=\frac{1}{2 \pi} \int_{-\infty}^{+\infty} d n_{2} \int_{-X_{2}}^{+X_{2}} d x_{2} \frac{\partial U_{1}}{\partial x_{2}} e^{-\imath n_{2} x_{2}}
$$

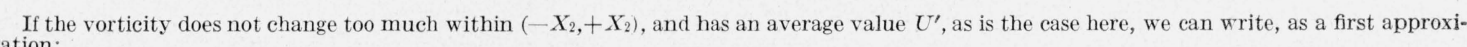

the factor before $U^{\prime}$ being unity.

$$
\int_{-\infty}^{+\infty} d n_{2} \iota n_{2} A_{1}\left(n_{2}\right)=\frac{1}{\pi} \int_{-\infty}^{+\infty} d n_{2} \frac{\sin n_{2} X_{2}}{n_{2}} \cdot U^{\prime}=U^{\prime},
$$


We notice that they are analogous in structural form, except that the transfer function contains a triple correlation

$$
q\left(\boldsymbol{n}, \boldsymbol{k}^{\prime \prime}\right) \equiv \iota n_{j} a_{i}(\boldsymbol{n}) a_{j}\left(\boldsymbol{k}^{\prime \prime}-\boldsymbol{n}\right) a_{i}\left(-\boldsymbol{k}^{\prime \prime}\right)+\text { conjugate }
$$

which is antisymmetrical: $q\left(\boldsymbol{n}, \boldsymbol{k}^{\prime \prime}\right)=-q\left(\boldsymbol{k}^{\prime \prime}, \boldsymbol{n}\right)$, while the production function contains a triple correlation involving the gradient of the mean motion and is not antisymmetrical. For the antisymmetrical function, we can replace

$$
\int_{k}^{\infty} d k^{\prime \prime} \int_{0}^{\infty} d n \text { by } \int_{k}^{\infty} d k^{\prime \prime} \int_{0}^{k} d n
$$

in agreement with the form of integrals given to $W(k, \infty)$ in $(9)$. For the production function, we can take the following form, because of its structual analogy with the transfer function:

$\Psi(k, \infty)=\kappa^{\prime \prime} \int_{k}^{\infty} d k^{\prime \prime} \sqrt{\frac{F\left(k^{\prime \prime}\right)}{k^{\prime \prime}}} \cdot$ (vorticity of turbulent motion $\cdot$ vorticity of mean motion)

Now the vorticity of the turbulent motion can be written in the usual form

$$
\left[2 \int_{0}^{k} d k^{\prime} k^{\prime 2} F\left(k^{\prime}\right)\right]^{\frac{1}{2}}
$$

while the vorticity of the mean motion is $U^{\prime}$. We can remark that transformation (13) does not apply to correlations involving the mean motion, and therefore the vorticity of the mean motion is written for its whole spectrum. After substitution for the vorticities, (14) becomes:

$$
\Psi(k, \infty)=\kappa^{\prime \prime} \int_{k}^{\infty} d k^{\prime \prime} \sqrt{\frac{F\left(k^{\prime \prime}\right)}{k^{\prime \prime}}}\left[2 \int_{k}^{\infty} d k^{\prime} k^{\prime 2} F\left(k^{\prime}\right) \cdot U^{\prime 2}\right]^{\frac{1}{2}} .
$$

Finally we shall integrate the convection term of (7) for the region outside the sphere of radius $k$. We can remark that the transfer, production, and convection contain, respectively, the triple correlations

$$
\begin{aligned}
& \text { `n } n_{j} a_{i}(\boldsymbol{n}) a_{j}(\boldsymbol{k}-\boldsymbol{n}) a_{i}(-\boldsymbol{k})+\text { conjugate } \\
& \text { in } n_{j} A_{i}(\boldsymbol{n}) a_{j}(\boldsymbol{k}-\boldsymbol{n}) a_{i}(-\boldsymbol{k})+\text { conjugate } \\
& \text { in } n_{j} a_{i}(\boldsymbol{n}) A_{j}(\boldsymbol{k}-\boldsymbol{n}) a_{i}(-\boldsymbol{k})+\text { conjugate. }
\end{aligned}
$$

By analogy with the expression (9) and (15) for the transfer and the production, we can formulate an expression for the convection function. However, we assume that the apparent viscosity is much smaller for the mean motion than for the turbulent motion, for the sufficiently large value of $k$ herewith considered; hence the convection function is negligible compared to $W(k, \infty)$ for sufficiently large $k$.

\section{Diffusion by Inhomogeneity of the Field}

Let us consider a case with the following simple conditions:

(a) The diffusion due to the inhomogeneity is primarily a large scale phenomenon, and hence the molecular diffusion can be neglected compared to the turbulent diffusion.

(b) The mean pressure is constant in time and space.

The difference of energy distribution in the space causes a flux of energy as a consequence of the exchange of matter. This flux of energy can be written as

$$
\text { flux of energy }=\kappa^{\prime \prime \prime} \int_{k}^{\infty} d k^{\prime \prime} \sqrt{\frac{F\left(k^{\prime \prime}\right)}{k^{\prime \prime}}} \cdot \frac{\partial}{\partial x_{2}}\left[\rho \int_{0}^{k} d k^{\prime} F\left(k^{\prime}\right)\right] \text {. }
$$

The first integral is the turbulent viscosity, the gradient is the gradient of the energy distribution, and $\kappa^{\prime \prime \prime}$ is a numerical constant to be determined by experiment. By taking the difference of the fluxes across opposite faces of the elementary space and dividing by $\rho$, we obtain the 
rate of decrease of energy by diffusion per unit mass and unit time equal to

$$
K(k, \infty)=\frac{\partial}{\partial x_{2}}\left[\kappa^{\prime \prime \prime} \int_{k}^{\infty} d k^{\prime \prime} \sqrt{\frac{F\left(k^{\prime \prime}\right)}{k^{\prime \prime}}} \cdot \frac{\partial}{\partial x_{2}} \int_{0}^{k} d k^{\prime} F\left(k^{\prime}\right)\right]
$$

Finally, if $k$ is chosen sufficiently large so that the range from 0 to $k$ includes practically all the energy gradient, we can write

Hence

$$
\frac{\partial}{\partial x_{2}} \int_{0}^{k} d k^{\prime} F\left(k^{\prime}\right) \cong \frac{\partial}{\partial x_{2}}\left(\frac{1}{2} \overline{u_{i}^{2}}\right)
$$

$$
K(k, \infty) \cong \frac{\partial}{\partial x_{2}}\left[k^{\prime \prime \prime} \int_{k}^{\infty} d k^{\prime \prime} \sqrt{\frac{F\left(k^{\prime \prime}\right)}{k^{\prime \prime}}} \frac{\partial}{\partial x_{2}}\left(\frac{1}{2} \overline{u_{i}^{2}}\right)\right]
$$

\section{Energy Spectrum of Turbulence}

In the equilibrium range the energy spectrum is determined by eq 8, which becomes, after substitution of (9), (12), (15), and (16),

$$
\begin{aligned}
\epsilon= & 2 \nu \int_{0}^{k} d k^{\prime} k^{\prime 2} F\left(k^{\prime}\right)+2 \kappa \int_{k}^{\infty} d k^{\prime \prime} \sqrt{\frac{F\left(k^{\prime \prime}\right)}{k^{\prime \prime}}} \int_{0}^{k} d k^{\prime} k^{\prime 2} F\left(k^{\prime}\right)+ \\
& \kappa^{\prime} \int_{k}^{\infty} d k^{\prime \prime} \sqrt{\frac{F\left(k^{\prime \prime}\right)}{k^{\prime \prime}}} \cdot U^{\prime 2} \zeta+\frac{\partial}{\partial x_{2}}\left[\kappa^{\prime \prime \prime} \int_{k}^{\infty} d k^{\prime \prime} \sqrt{\frac{F\left(k^{\prime \prime}\right)}{k^{\prime \prime}}} \frac{\partial}{\partial x_{2}}\left(\frac{1}{2} \overline{u_{i}^{2}}\right)\right]
\end{aligned}
$$

with

$$
\zeta=\left\langle\frac{\kappa^{\prime \prime}}{\kappa^{\prime}}\left[\frac{2 \int_{0}^{k} d k^{\prime} k^{\prime 2} F\left(k^{\prime}\right)}{U^{\prime 2}}\right] \text {, strong resonance }\right\}
$$

We shall study the energy spectrum for the following subranges: (a) nonviscous subrange with large $U^{\prime}$ and strong resonance, (b) nonviscous subrange with small $U^{\prime}$ and no resonance, and (c) viscous subrange with arbitrary $U^{\prime}$ and arbitrary resonance.

(a) In the nonviscous subrange of the spectrum, if there is a strong resonance and if $U^{\prime}$ is large, along with sufficiently small $k$, we have

$$
2 \int_{0}^{k} d k^{\prime} k^{\prime 2} F\left(k^{\prime}\right) \ll U^{\prime 2}
$$

thus the production function is predominant, and all other functions are negligible. In this way eq (17) reduces to

$$
\frac{\epsilon}{\kappa^{\prime \prime} U^{\prime}}=\int_{k}^{\infty} d k^{\prime \prime} \sqrt{\frac{F\left(k^{\prime \prime}\right)}{k^{\prime \prime}}}\left[2 \int_{0}^{k} d k^{\prime} k^{\prime 2} F\left(k^{\prime}\right)\right]^{\frac{1}{2}}
$$

and has the solution $F=\left(\epsilon / \kappa^{\prime \prime} U^{\prime}\right) k^{-1}$ to be obtained simply by introducing the new variable $g^{2}=2 \int_{0}^{k} d k^{\prime} k^{\prime 2} F\left(k^{\prime}\right)$. If the diffusion term is small, but not negligible, then as a first approximation, the solution is

where

$$
F=\frac{\epsilon}{\kappa^{\prime \prime} U^{\prime}} k^{-1}\left(1-k_{r} / k\right)
$$

$$
k_{\tau}=\frac{\kappa^{\prime \prime}}{\epsilon} \frac{\partial}{\partial x_{2}}\left[\left(\frac{\epsilon}{\kappa^{\prime \prime} U^{\prime 2}}\right)^{\frac{1}{2}} \frac{\partial}{\partial x_{2}}\left(\frac{1}{2} \overline{u_{i}^{2}}\right)\right]
$$

and $k_{\tau} / k$ is assumed to be small compared to unity. 
(b) In the nonviscous subrange, if there is no resonance and $U^{\prime}$ is small, along with sufficiently large $k$, we have

$$
2 \int_{0}^{k} d k^{\prime} k^{\prime 2} F\left(k^{\prime}\right) \gg U^{\prime 2}
$$

Thus the production function and the diffusion function are small, although not negligible compared to the transfer function, and eq (17) gives the following solution as a first approximation:

$$
F=\left(\frac{8 \epsilon}{9 \kappa}\right)^{2 / 3} k^{-5 / 3}\left[1-\left(k_{p} / k\right)^{4 / 3}\right]^{2 / 3},
$$

where

$$
k_{p}^{4 / 3}=\left(\frac{3}{8} \frac{\kappa^{\prime 3}}{\kappa} \frac{1}{\epsilon^{2}}\right)^{1 / 3} U^{\prime 2}+\left(\frac{3}{8} \frac{\kappa^{\prime \prime \prime}}{\kappa} \frac{1}{\epsilon^{3}}\right)^{1 / 3} \frac{\partial}{\partial x_{2}}\left[\epsilon^{1 / 3} \frac{\partial}{\partial x_{2}}\left(\frac{1}{2} \overline{u_{i}^{2}}\right)\right]
$$

and $k_{p} / k$ is assumed to be small compared to unity. The spectrum

$$
F=\left(\frac{8 \epsilon}{9 \kappa}\right)^{2 / 3} k^{-5 / 3}
$$

or the homogeneous and isotropic turbulence is a particular case corresponding to $k_{p}=0$.

For the reasons incorporated already in the conditions under which the respective laws $k^{-1}$ and $k^{-5 / 3}$ are obtained, it is to be understood that the former law refers usually to larger eddies than does the latter one. Depending upon specific conditions, a spectrum may show clearly a portion representative of one of the laws and not the other, or may show clearly two portions representative of both laws successively. In the latter case, the primary motion possesses a pattern in resonance with the secondary motion. As the result of the resonance, the production function is first larger than the transfer function at small $k$, hence the spectrum shows a $k^{-1}$ law. Afterwards, as $k$ increases, the production function becomes smaller than the transfer function, while $U^{\prime}$ is not large enough to compensate, hence the spectrum changes over to a $k^{-5 / 3}$ law. The ranges of validity of the laws depend upon the ranges of predominance either by the production function or by the transfer function. For the viscous subrange, which we shall study in (c), the eddies are much smaller than the nonviscous subrange. Consider the case where we have a $k^{-1}$ law, thus $k$ is small and $U^{\prime}$ is large. Suppose that the Reynolds number is not sufficiently large, so that the viscous dissipation may become so important as to alter the tail portion of the $k^{-1}$ spectrum. Under such circumstances, for increasing wave numbers, the $k^{-1}$ law may pass over directly to the $k^{-7}$ law, without a noticeable portion of the $k^{-5 / 3}$ law. For very small Reynolds numbers, a larger part of the tail portion or even the whole of the $k^{-1}$ law may be affected, as could be observed in the turbulent flow in the close vicinity of a wall.

(c) In the viscous subrange, the turbulent friction of turbulent viscosity

$$
\kappa \int_{k}^{\infty} d k^{\prime \prime} \sqrt{F\left(k^{\prime \prime}\right) / k^{\prime \prime}}
$$

is much smaller than the molecular friction. By differentiating (17) with respect to $k$, and by neglecting the turbulent viscosity compared to the molecular viscosity, we have

$$
0 \simeq 2 \nu k^{2} F-\frac{\kappa \epsilon}{\nu} \sqrt{\frac{F^{\prime}}{k^{3}}}\left[1+\frac{\kappa^{\prime}}{\kappa} \frac{\nu}{\epsilon} U^{\prime 2} \zeta_{\infty}+\frac{\kappa^{\prime \prime \prime}}{\kappa} \frac{\nu}{\epsilon} \frac{\partial^{2}}{\partial x_{2}^{2}}\left(\overline{u_{i}^{2}} / 2\right)\right]
$$

where $\zeta_{\infty}$ is obtained by putting $k=\infty$ in expression (17a).

The solution follows:

$$
F=\left(\frac{\kappa \epsilon}{2 \nu^{2}}\right)^{2} k^{-7}\left[1+\frac{\kappa^{\prime}}{\kappa} \frac{\nu}{\epsilon} U^{\prime 2} \zeta_{\infty}+\frac{\kappa^{\prime \prime \prime}}{\kappa} \frac{\nu}{\epsilon} \frac{\partial^{2}}{\partial x_{2}^{2}}\left(\overline{u_{i}^{2}} / 2\right)\right] \text {. }
$$

For homogeneous and isotropic turbulence, the above formula degenerates to the Heisenberg formula [6]

$$
F=\left(\frac{\kappa \epsilon}{2 \nu^{2}}\right)^{2} k^{-7}
$$




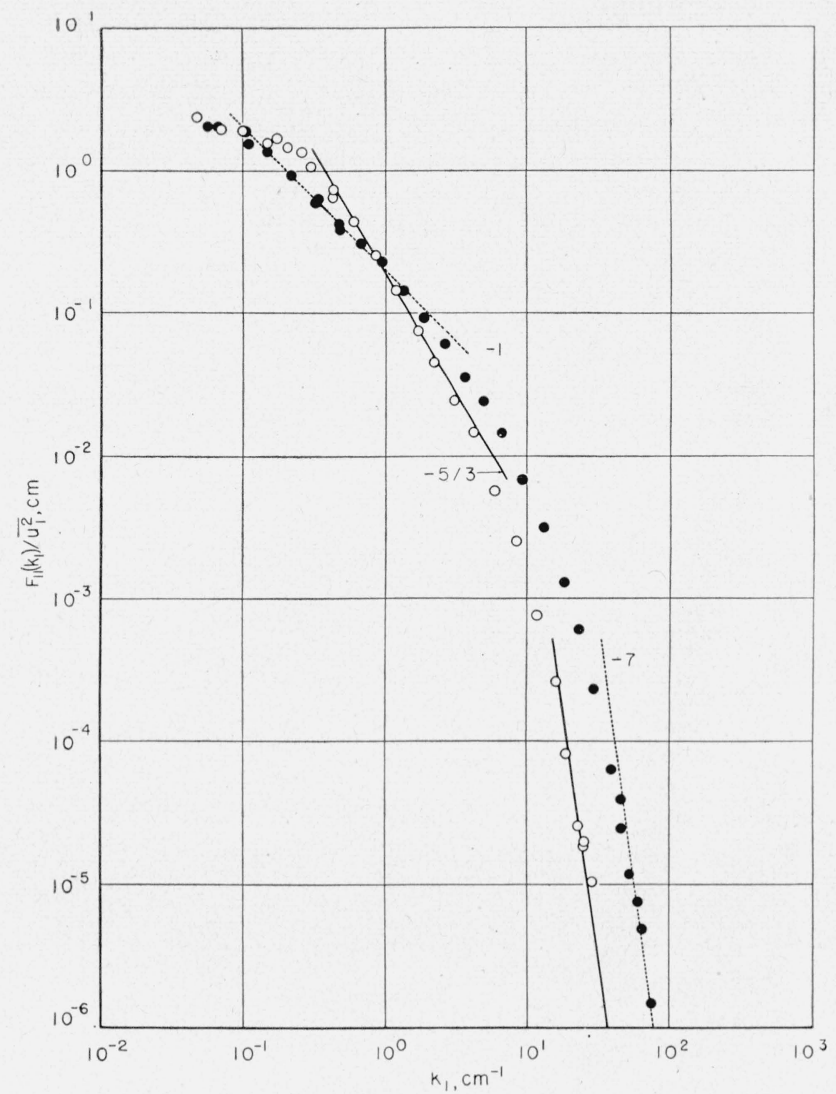

FIGURE 1. Energy spectrum in a turbulent boundary layer.

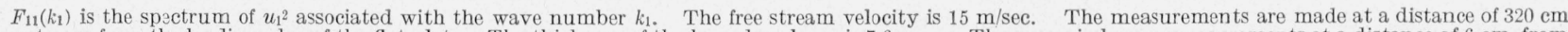

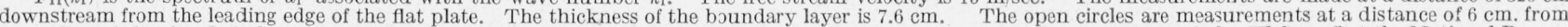

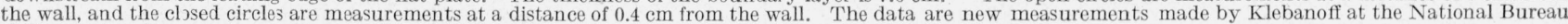
of Standards. Some early results are published in reference [11].

The $k^{-7}$ law follows from the assumption of the Heisenberg expression for the transfer function. We see that the eddies in the viscous subrange of the spectrum are not effected by any shear.

We shall compare (see fig. 1) the above results with the experimental investigations in a turbulent boundary layer made by P. S. Klebanoff and Z. W. Diehl [11]. It is to be remarked that the available measurements give the spectral function for $\overline{u_{i}^{2}} / 2$ in the one-dimensional analysis of the wave-number space, while the spectral function in the theory refers to the energy $\overline{u_{i}^{2}} / 2$ for the wave-number magnitude in the three dimensional analysis of the wavenumber space. Assuming that the power laws for sufficiently large $k$ remains unaltered by the transformation between the two kinds of spectral functions for the cases considered, we see that the laws $k^{-1}, k^{-5 / 3}, k^{-7}$ agree with experiments respectively for the following cases:

(a) Near the wall where the average scale of the primary motion is small, there is strong resonance between the primary motion and the turbulent motion. If, moreover,

$$
U^{\prime 2} \gg 2 \int_{0}^{k} d k^{\prime} k^{\prime 2} F\left(k^{\prime}\right)
$$

in the nonviscous subrange, the production of turbulence predominates, and the spectral law is $k^{-1}$.

(b) Far away from the wall, and still not close to the rough edge of the boundary layer, the average scale of the primary motion is large, and the resonance is negligible. If, moreover,

$$
U^{\prime 2} \ll 2 \int_{0}^{k} d k^{\prime} k^{\prime 2} F\left(k^{\prime}\right)
$$

in the nonviscous subrange, the transfer of energy among the eddies predominates, and the spectral law is $k^{-5 / 3}$. 
(c) In the viscous subrange, the eddies are under no circumstance effected by the shear. Therefore, everywhere in the boundary layer the power law $k^{-7}$ of Heisenberg is reproduced, although this law is not expected to continue to infinite wave numbers.

If we consider as before a turbulent flow with a mean velocity in the $x_{1}$ direction, with a gradient $\partial U_{1} / \partial x_{2}$, and write energy equations of the type (7) and (7a), we notice that the production function will be absent in the energy equations for the $u_{2}$ and $u_{3}$ components. Therefore, the spectra of $u_{2}$ and $u_{3}$ will not show any $k^{-1}$ law which is characteristic of the production function.

\section{References}

[1] Th. v. Kármán and L. Howarth, Proc. Roy. Soc. London A $164,192(1938) ;$ J. M. Burgers, Proc. Acad. Sci. Amsterdam 8, 122, 248, 393, 718, 732 (1950).

[2] J. M. Burgers, Verhand. Kon. Nederl. Akademie v. Wetenschappen (le sect.) 1\%, 18 (1939); Advances in Appl. Mech. 1, 189 (1948).

[3] L, Onsager, Nuovo Cimento, Supplemento 6, ser, 9, No. 2, 279 (1949); Phys. Rev. 68, 286 (1945).

[4] A. M. Obukhoff, Bul. Acad. URSS, Ser. Geogr. Geophys. 453 (1941); Doklady 32, 19 (1941).

[5] C. F. v. Weiszäcker, Z. Phys. 124, 614 (1948).
[6] W. Heisenberg, Z. Phys. 124, 628 (1948).

[7] A. N. Kolmogoroff, Doklady 30, 301 (1941); 32, 16 (1941).

[8] L. Prandtl, Z. Angew. Math. Mech. 5, 136 (1925).

[9] J. J. Boussinesq, Théorie de l'écoulement tourbillonnant et tumultueux des liquides, Paris (1897).

[10] C. M. Tchen, J. Chem. Phys, 20, 214 (1952).

[11] P. S. Klebanoff and Z. W. Diehl, NACA Tech. Notes 2475 (1951).

Washington, August 23, 1951. 